\title{
Life-threatening oral mucositis following chemotherapy in a pediatric patient
}

Kaoru Tsuboi ${ }^{1}$, Norihiko Tsuboi $^{1}$, Kenichi Sakamoto ${ }^{2}$, Akira Takebayashi ${ }^{2}$, Daisuke Tomizawa $^{3}$, Nao Nishimura ${ }^{1}$, and Satoshi Nakagawa ${ }^{1}$

${ }^{1}$ National Center for Child Health and Development Hospital

${ }^{2}$ Children's Cancer Center, National Center for Child Health and Development

${ }^{3}$ Tokyo Medical and Dental University

March 16, 2021

\begin{abstract}
Pediatric patients undergoing chemotherapy may present upper airway obstruction due to severe oral mucositis. Although reversible, its clinical course correlates with the course of neutropenia and may be complicated. Thus, airway management in these patients must be determined on an individual case basis.
\end{abstract}

Title page

Title

Life-threatening oral mucositis following chemotherapy in a pediatric patient

Authors

Kaoru Tsuboi ${ }^{1}$, M.D. Email: shimizu-k@ncchd.go.jp

Norihiko Tsuboi ${ }^{1}$, M.D. Email: tsuboi-n@ncchd.go.jp

Kenichi Sakamoto², M.D. Email:sakamoto-k@ncchd.go.jp

Akira Takebayashi², M.D. Email:takebayashi-a@ncchd.go.jp

Daisuke Tomizawa² ${ }^{2}$ M.D., Ph.D. Email: tomizawa-d@ncchd.go.jp

Nao Nishimura ${ }^{1}$, M.D. Email: nishimura-n@ncchd.go.jp

Satoshi Nakagawa ${ }^{1}$, M.D. Email:nakagawa-s@ncchd.go.jp

1 Department of Critical Care Medicine, National Center for Child Health and Development. 2-10-1 Okura, Setagaya-ku, Tokyo, Japan

2 Children's Cancer Center, National Center for Child Health and Development. 2-10-1 Okura, Setagaya-ku, Tokyo, Japan

\section{Corresponding Author}

Kaoru Tsuboi

mailing address: Department of Critical Care Medicine, National Center for Child Health and Development. 2-10-1 Okura, Setagaya-ku, Tokyo, Japan 
Email address: shimizu-k@ncchd.go.jp

Fax: +81-3-3416-2222

Telephone number: +81-3-3416-0181

\section{Abstract}

Pediatric patients undergoing chemotherapy may present upper airway obstruction due to severe oral mucositis. Although completely reversible, its clinical course correlates with the course of neutropenia and may be complicated. Thus, airway management in these patients must be determined on an individual case basis.

\section{Keywords}

Mucositis, chemotherapy, upper airway obstruction, neutropenia, pediatric

\section{Main text}

\section{Introduction}

Mucositis is a common adverse effect in pediatric patients receiving chemotherapy for hematological malignancies $^{1}$. However, severe mucositis leading to upper airway obstruction is rare and literature regarding this topic is scarce. It is an impending oncologic emergency and clinicians should be aware of the clinical course and management of this life-threatening complication.

\section{Case Presentation}

A 10-year-old, $34 \mathrm{~kg}$ female with no significant medical history was admitted with recently diagnosed acute myeloid leukemia. She was treated with etoposide $150 \mathrm{mg} / \mathrm{m}^{2} /$ dose on days $1-5$, cytarabine $200 \mathrm{mg} / \mathrm{m}^{2} /$ dose on days 6-12, mitoxantrone $5 \mathrm{mg} / \mathrm{m}^{2} /$ dose on days 6-10, and triple intrathecal therapy on day 6 as induction therapy (JPLSG AML-05 protocol ${ }^{2}$ ). Following chemotherapy, her course was complicated by continued febrile neutropenia and oral mucositis. Broad spectrum antibiotics including meropenem, teicoplanin, and caspofungin with granulocyte colony-stimulating factor (G-CSF) was initiated. As multiplex polymerase chain reaction proved positive for Herpes simplex viral infection, acyclovir was added. She complained of severe throat pain and dysphagia due to worsening mucositis and required continuous infusion of morphine.

On day 24, the patient complained of increasing throat pain and difficulty breathing. She was found sitting up in bed, leaning forward, drooling. Pan-inspiratory stridor and mild effort of breathing was observed on physical examination. She was alert with her vital signs otherwise stable. Examination on nasolaryngoscopy revealed extensive desquamation of the oropharyngeal mucosa and multiple, confluent ulcerative lesions with significant edema extending down to the arytenoid cartilage (Fig. 1). The patient was taken to the operating room for elective intubation with otolaryngology team on standby for tracheostomy placement. A size 5.0 $\mathrm{mm}$ internal diameter endotracheal tube was successfully inserted by video laryngoscopy with anesthesia maintaining spontaneous ventilation.

The patient was then transported to the pediatric intensive care unit. As infection being considered as an aggravating factor, antimicrobiotic agents and G-CSF was continued, and granulocyte transfusion from paternal donor was attempted.

On day 28, she developed significant spontaneous bleeding from the gingiva and the oropharyngeal ulcers, resulting in hemorrhagic shock. Platelet transfusions and vasoconstrictors were warranted for resuscitation. Nasolaryngoscopy revealed further aggravation of the ulcerative lesions with active oozing and worsening edema of the oropharyngeal tissues showing complete obstruction of the retropharyngeal space. Packing of the oropharyngeal space was performed as electrocoagulation of bleeding vessels failed to control bleeding.

Surgical tracheostomy was considered taking the above finding into account. After thorough discussion with the oncologists and the otolaryngologists a decision was made to wait for resolution of neutropenia considering the elevated risk of procedure in the nadir phase and expected improvement of mucositis following recovery of neutrophil counts. 
On day 29, absolute neutrophil count exceeded $500 / \mu \mathrm{L}$ and G-CSF was discontinued. Her bleeding was well controlled. On day 31, defervescence was achieved. On day 32, improvement of the ulcerative lesions and airway swelling was confirmed on nasolaryngoscopy. Adequate leakage around the endotracheal tube was verified. The patient was able to cough, swallow efficiently requiring minimum ventilatory support. Extubation was successful on first attempt. Repeated blood cultures obtained throughout her course were found to be negative. Her course after extubation was uneventful.

\section{Discussion}

Oral mucositis $(\mathrm{OM})$ is a common adverse event in patients receiving chemotherapy. Its clinical manifestation ranges from mild erythema and soreness to extreme pain and ulceration, significantly affecting patient's quality of life ${ }^{3}$. Specific drug therapy has been associated with an increased risk of developing mucositis; etoposide is excreted in the saliva which increases its oral toxicity ${ }^{1}$. In rare cases, severe OM can result in significant edema of the upper airway leading to airway obstruction ${ }^{4}$.

Pediatric patients are more prone to develop OM with increased severity ${ }^{5}$. Its prevalence is reported to exceed $90 \%$ among children under 12 years old receiving chemotherapy for hematological malignancies compared with $40 \%$ in the adult counterparts ${ }^{1}$. This has been attributed to the greater number of mitoses in the basal epithelium in younger patients, making the epithelial cells more vulnerable to cytotoxic effects ${ }^{6}$. The duration of $\mathrm{OM}$ in the pediatric population tends to be shorter reflecting their greater healing capacity ${ }^{6}$.

Pediatric patients with severe OM are at greater risk of airway compromise due to their narrower airway ${ }^{6}$. The exact prevalence of upper airway complications in the pediatric patients following chemotherapy is unknown. One study reported that $10.5 \%$ of the pediatric patients following bone marrow transplantation required mechanical ventilation, of which upper airway obstruction due to mucositis accounted for $13 \%{ }^{7}$.

Typically, OM develops 10-14 days after initiation of chemotherapy and heals within 2-4 weeks, consistent with the clinical course of neutropenia ${ }^{8}$. Neutropenia has been reported to play a key role in the development of OM. Severity of OM is associated with the degree of neutropenia, and resolution coincides with granulocyte recovery ${ }^{8}$. This is assumed to be a result of neutropenia leading to impaired mucosal defence and repair ${ }^{8}$.

Management of OM is crucial as it provides a portal for potentially life-threatening infection ${ }^{8}$. Previous studies have shown that the risk of infection increases with increasing grade of $\mathrm{OM}^{8}$. As most patients with $\mathrm{OM}$ develop neutropenic fever, broad spectrum beta-lactam with combination of antifungal and antiviral agents is commonly used as a standard treatment to cover gram-negative bacteria, Coagulase-negative Streptococci ,Streptococcus viridans, Candida albicans and Herpes simplex ${ }^{6,9,10}$. However, fever remains unexplained in 30-50\% of neutropenic patients with no evidence of infection ${ }^{11}$. Furthermore, fever persists for 4-5 days or even longer in approximately $30 \%$ of cases despite adequate microbial treatment directed at bacteria and fungi ${ }^{11}$. In these cases, fever may not be necessarily related to infection and may be a manifestation of the inflammatory process contributed by the mucositis itself ${ }^{9,11}$. In a report examining patients receiving chemotherapy for treatment of acute leukemia, inflammation was shown to be correlated with the occurrence of mucositis. The inflammatory response is elicited by formation of reactive oxygen species due to DNA damage of the epithelial cells, followed by amplification of proinflammatory and inflammatory cytokine release triggered by various microbial motifs (Pathogen-associated molecular patterns) released from invading microorganisms and damaged tissues (Damage-associated molecular patterns) through the distorted mucosal barrier, manifesting systemic fever and further tissue damage ${ }^{11}$.

In the current case, the patient remained febrile despite treatment with broad spectrum antibiotics, antifungal and antiviral agents lacking evidence of infection. Regarding her prompt defervescence coinciding with recovery of mucositis, her fever may have been a manifestation of the inflammation of mucositis, irrelevant of infection. Currently no guideline is provided regarding the use of G-CSF on mucositis due to insufficient evidence $^{12}$. Yet, the possibility of infection could not be discarded in this circumstance and treatment with broad-spectrum antimicrobials combined with G-CSF was continued.

In the current case, the patient was successfully extubated following recovery of mucositis but required 
prolonged mechanical ventilation. Determination whether to perform tracheostomy in this case was difficult considering its completely reversible pathology.

Airway management with intubation possesses a significant risk of complete airway obstruction and death in case of accidental extubation, mandating deep sedation (paralysis may be considered) and prolonged mechanical ventilation which significantly delays patient recovery.

Placement of a tracheostomy provides a secure airway and enables safe management of the patient with further advantages of improved patient comfort, reduced sedative requirements and early patient recovery. Although surgical tracheostomy in the presence of severe immunosuppression and thrombocytopenia is not a contraindication, it may be associated with increased risk of surgical complications. Studies regarding this topic are scarce, especially in the pediatric population and do not support a recommendation ${ }^{13-15}$.

Thus, airway management in these patients must be determined prudently regarding the expected duration of neutropenia, weighting the risks and benefits on an individual case basis.

\section{Conclusion}

Pediatric patients undergoing chemotherapy may require intubation and mechanical ventilation due to airway compromise as a complication. The clinical course of oral mucositis is consistent with the course of neutropenia and may require prolonged intubation. Determination whether to perform tracheostomy in these circumstances is difficult due to the essentially reversible pathology of this complication. Thus, airway management of these patients should be determined on an individual case basis.

\section{Acknowledgement}

The authors gratefully acknowledge the assistance of Dr. Noriko Morimoto of the department of otolaryngology, National Center for Child Health and Development for providing the laryngoscopy images.

\section{Disclosure}

None.

\section{Conflict of Interest}

The authors have no conflict of interest to declare.

\section{Author Contributions}

K.T. drafted and revised the initial manuscript; N.T., K.S., A.T., D.T., N.N., S.N. critically revised the manuscript for important intellectual content. All authors read and approved the final manuscript.

\section{Ethical approval}

Informed consent was obtained from the patient and her parents for publication of this case report. Approval of the institutional ethics committee was obtained in advance to submission.

\section{References}

1. Damascena LCL, de Lucena NNN, Ribeiro ILA, Pereira TL, Lima-Filho LMA, Valença AMG. Severe Oral Mucositis in Pediatric Cancer Patients: Survival Analysis and Predictive Factors. Int J Environ Res Public Health. 2020;17:1235

2. Tomizawa D, Tawa A, Watanabe $\mathrm{T}$ et al. Excess treatment reduction including anthracyclines results in higher incidence of relapse in core binding factor acute myeloid leukemia in children. Leukemia. 2013;27:24136

3. Kusiak A, Jereczek-Fossa BA, Cichońska D, Alterio D. Oncological-Therapy Related Oral Mucositis as an Interdisciplinary Problem-Literature Review. Int J Environ Res Public Health. 2020;17:2464 
4. Chaimberg KH, Cravero JP. Mucositis and airway obstruction in a pediatric patient. Anesth Analg. 2004;99:59-61

5. Walsh LJ. Clinical assessment and management of the oral environment in the oncology patient. Aust Dent J. 2010;55:66-77

6. Raber-Durlacher JE, Weijl NI, Abu Saris M, de Koning B, Zwinderman AH, Osanto S. Oral mucositis in patients treated with chemotherapy for solid tumors: a retrospective analysis of 150 cases. Support Care Cancer. 2000;8:366-371

7. Drew B, Peters C, Rimell F. Upper airway complications in children after bone marrow transplantation. Laryngoscope. 2000;110:1446-1451.

8. McCann S, Schwenkglenks M, Bacon P, et al. The Prospective Oral Mucositis Audit: relationship of severe oral mucositis with clinical and medical resource use outcomes in patients receiving high-dose melphalan or BEAM-conditioning chemotherapy and autologous SCT. Bone Marrow Transplant. 2009;43:141-147

9. Blijlevens NMA, Logan RM, Netea MG. Mucositis: from febrile neutropenia to febrile mucositis. Journal of Antimicrobial Chemotherapy, 2009;63:i36-i40

10. Blijlevens NM, Donnelly JP, de Pauw BE. Empirical therapy of febrile neutropenic patients with mucositis: challenge of risk-based therapy. Clin Microbiol Infect. 2001;7:47-52

11. van der Velden WJ, Herbers AH, Netea MG, Blijlevens NM. Mucosal barrier injury, fever and infection in neutropenic patients with cancer: introducing the paradigm febrile mucositis. Br J Haematol. 2014;167:441452

12. Raber-Durlacher JE, von Bültzingslöwen I, Logan RM, et al. Systematic review of cytokines and growth factors for the management of oral mucositis in cancer patients. Support Care Cancer. 2013;21:343-355

13. Manna SS, Malik R, Douthwaite A, Vaidya S. Surgical tracheostomy in children with malignancy receiving intensive chemotherapy: A case series and review of literature. Pediatr Blood Cancer. 2020;67:e28105

14. Raimondi N, Vial MR, Calleja J, et al. Evidence-based guidelines for the use of tracheostomy in critically ill patients. J Crit Care. 2017;38:304-318

15. Blot F, Nitenberg G, Guiguet M, et al. Safety of tracheotomy in neutropenic patients: a retrospective study of 26 consecutive cases. Intensive Care Med. 1995;21:687-690

\section{Figure captions}

Fig.1

Nasolarygoscopy revealed significantly swollen, erythematous arytenoid cartilages with extensive desquamation. 


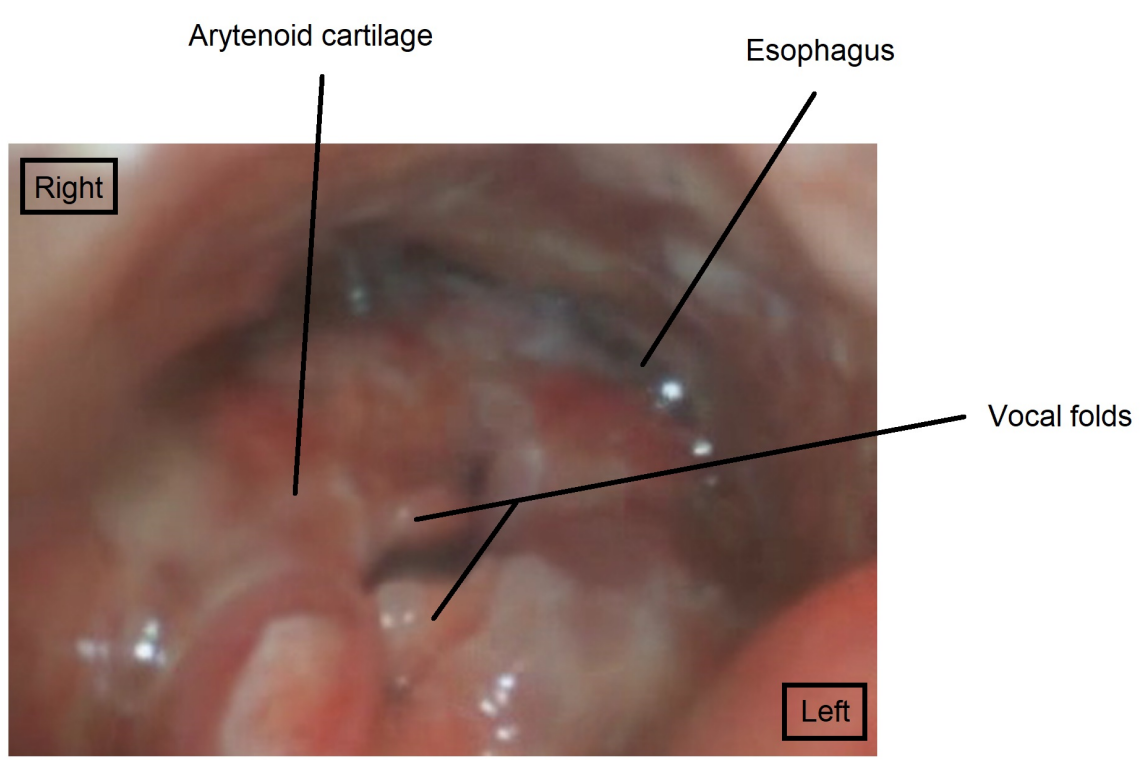

